\title{
Reference-Based MRI
}

\author{
Lior Weizman ${ }^{\text {a) }}$ \\ Department of Electrical Engineering, Technion - Israel Institue of Technology, \\ Israel \\ Yonina C. Eldar \\ Department of Electrical Engineering, Technion - Israel Institue of Technology, \\ Israel \\ Dafna Ben Bashat \\ Functional Brain Center, Tel Aviv Sourasky Medical Center, \\ Sackler Faculty of Medicine and Sagol School of Neuroscience, Tel Aviv University, \\ Israel
}

(Dated: 28 August 2017)

a) Corresponding author, e-mail:weizmanl@tx.technion.ac.il, phone:+972-4-8291724 
Purpose: In many clinical MRI scenarios, existing imaging information can be used to significantly shorten acquisition time or to improve Signal to Noise Ratio (SNR). In this paper the authors present a framework for fast MRI by exploiting a reference image (FASTMER).

Methods: The proposed approach utilizes the possible similarity of the reference image that exists in many clinical MRI imaging scenarios. Such scenarios include similarity between adjacent slices in high resolution MRI, similarity between various contrasts in the same scan and similarity between different scans of the same patient. The authors take into account that the reference image may exhibit low similarity with the acquired image and develop an iterative weighted approach for reconstruction, which tunes the weights according to the degree of similarity.

Results: Experimental results demonstrate the performance of the method in three different clinical MRI scenarios: SNR improvement in high resolution brain MRI, exploiting similarity between T2-weighted and fluid-attenuated inversion recovery (FLAIR) for fast FLAIR scanning and utilizing similarity between baseline and follow-up scans for fast follow-up. Results outperform reconstruction results of existing state-of-the-art methods.

Conclusions: The authors present a method for fast MRI by exploiting a reference image. The method is based on an iterative reconstruction approach that supports cases in which similarity to the reference scan is not guaranteed, which enables the applicability of the method to a variety of MRI applications. Thanks to the existence of reference images in various clinical imaging scenarios, the proposed framework can play a major part in improving reconstruction in many MR applications.

Keywords: Rapid MR, Compressed Sensing, Reference based MRI 


\section{INTRODUCTION}

Magnetic resonance imaging (MRI) data is sampled in the spatial Fourier transform (a.k.a. k-space) of the object under investigation. In many cases, the k-space is sampled below the Nyquist rate due to constraints in the implementation of the k-space trajectory that control the sampling pattern (e.g., acquisition duration and smoothness of gradients). Mostly, prior assumptions on the nature of the data are taken into account in the reconstruction process, to overcome imaging artifacts due to insufficient sampling. We can roughly divide MRI reconstruction approaches from undersampled k-space into two families: singleand multiple-image based recovery.

The first family of methods exploits prior assumptions on a single MR image, in order to improve its reconstruction from undersampled data. Since the introduction of Compressed Sensing $(\mathrm{CS})^{1-4}$ to the field of $\mathrm{MRI}^{5}$, many MRI reconstruction approaches exploit the fact that MR images are highly compressible, by formalizing the image reconstruction problem as an $\ell_{1}$ minimization problem. Wavelet transform sparsity has been widely used as a sparsifying transform for brain MRI. Total Variation (TV) has been used for MR images which are sparse in the image domain, such as angio-MRI ${ }^{5,6}$. Other approaches focus on learning the sparsifying transform or using a dictionary developed exclusively for $\mathrm{MRI}^{7-9}$.

The second family of techniques exploit similarity to a single reference image or within a series of MRI images. In Table I we present a concise review of prior art in methods that exploit reference images to speed-up acquisition or to improve image reconstruction. Most algorithms were applied to dynamic or Diffusion MRI, where multiple images are acquired at a single imaging session. This allows the exploitation of similarity along the temporal dimension, assuming that only parts of the field-of-view (FOV) change over time. State-of-the-art approaches exploit the temproal similarity in a generalized reconstruction scheme $^{10-14}$, in a Bayesian-based approach ${ }^{15}$, in a CS framework ${ }^{16-20}$, or by enforcing lowrank reconstruction ${ }^{21-23}$.

Additional works focus on structural MRI. Some use a-priori information to improve reconstruction of single-contrast $\mathrm{MRI}^{24-27}$. In multiple-contrast MRI, structural similarity between different contrasts in the same scan is assumed and can be used to enhance reconstruction ${ }^{28-31}$. A few works have been published on exploiting similarity in longitudinal MRI, where images were acquired at different time points ${ }^{32-34}$. Finally, the use of 
reference images to improve reconstruction has also been tested in other imaging modalities, such as Magnetic Resonance Spectroscopy (MRS) ${ }^{10,35}$, Positron Emission Tomography $(\mathrm{PET})^{36-38}$ and X-Ray Computed Tomography (X-Ray CT) ${ }^{39}$.

Taking a deeper look into the rightmost column of Table I, we observe that multipleimage based reconstruction is application specific; since similarity between multiple images takes on different forms, a separate reconstruction approach was developed for each MRI application, exploiting its specific nature. No general sampling and reconstruction scheme which fits a variety of multiple-image MRI applications has been developed so far. Moreover, most of the methods rely on the assumption that there is substantial similarity between the images in the series (e.g. dynamic imaging), in the image or in some transform domain. Assuming similarity of intensity between the reference image and the current scan may not always be valid (e.g. when a different imaging contrast is used as a reference or due to misalignment between images) and therefore may lead to undesired reconstruction results.

Recently, we introduced an iterative approach for sampling and reconstruction exploiting similarity across multiple MRIs of the same patient ${ }^{40}$. We considered the acquisition of a follow-up MRI, given the baseline scan of the same patient. We took into account that baseline and follow-up images may not exhibit similarity and developed an iterative weighted mechanism that adjusts the reconstruction parameters and the sampling locations during real-time scanning. While the idea of optimizing the sampling locations has also been proposed by others ${ }^{41}$, it suffers from practical difficulties due to the necessity to solve many computationally heavy $\ell_{1}$ minimization problems during the acquisition process.

In a related work ${ }^{42}$, we have shown that enforcing similarity between adjacent, low SNR, thin MRI slices, can lead to significant improvement in SNR that obviates the need for multiple excitations for high SNR. Our approach is based on modifying the RF pulse signal for different weighting of the thin slices in the acquired data, which might be difficult in practical implementation in existing MRI hardware.

In a recent conference paper we presented the initial concept of applying a reference-based approach for multiple MRI applications ${ }^{43}$. The conference paper includes an earlier version of the model described in this paper, with preliminary results obtained via retrospective 2-dimensional random sampling. 
TABLE I Summary of related methods that exploit a reference image for fast MRI. Our proposed method is the last in the table. Abbreviations used in the table are: DCE: dynamic contrast enhanced, CS: compressed sensing, DWI: diffusion weighted imaging, TV: total variation, fMRI: functional MRI

\begin{tabular}{|c|c|c|}
\hline Author & Description & $\begin{array}{l}\text { Imaging Application } \\
\text { Tested }\end{array}$ \\
\hline Liang et al. ${ }^{44}$ & $\begin{array}{l}\text { Exploiting temporal similarity in dynamic MRI using generalized scheme } \\
\text { imaging }\end{array}$ & $\begin{array}{l}\text { Dynamic MRI (dynamic } \\
\text { T1-weighted and diffusion } \\
\text { MRI) }\end{array}$ \\
\hline Hanson et al. ${ }^{11}$ & $\begin{array}{l}\text { Exploiting two high resolution reference images to improve dynamic imaging } \\
\text { in a generalized scheme }\end{array}$ & Dynamic MRI (DCE MRI) \\
\hline Hess et al. ${ }^{12}$ & $\begin{array}{l}\text { Exploiting reference image for generation of basis functions, used to improve } \\
\text { dynamic MRI }\end{array}$ & $\begin{array}{l}\text { Dynamic MRI (MR } \\
\text { angiography) }\end{array}$ \\
\hline Tsao et al. ${ }^{32}$ & $\begin{array}{l}\text { Incorporating reference image and prior on changed regions for improved } \\
\text { reconstruction }\end{array}$ & Longitudinal MRI \\
\hline Tsao et al. ${ }^{45}$ & $\begin{array}{l}\text { Exploiting spatiotemporal correlations for dynamic MRI (training-based } \\
\text { approach) }\end{array}$ & $\begin{array}{l}\text { Dynamic MRI (cardiac } \\
\text { imaging) }\end{array}$ \\
\hline Lustig et al. ${ }^{16}$ & $\begin{array}{l}\text { Random sampling in k-t space, reconstruction based on wavelet-Fourier } \\
\text { sparsity }\end{array}$ & $\begin{array}{l}\text { Dynamic MRI (cardiac } \\
\text { imaging) }\end{array}$ \\
\hline Haldar et al. ${ }^{46}$ & Using anatomical priors to improve SNR via penalized ML & Single-contrast MRI \\
\hline Lang et al. ${ }^{17}$ & Exploiting similarity to a reference image in a CS framework & Dynamic MRI (brain DCE) \\
\hline Gamper et al. ${ }^{18}$ & Exploiting sparsity in the $\mathrm{x}-\mathrm{f}$ space for dynamic MRI & $\begin{array}{l}\text { Dynamic MRI (cardiac } \\
\text { imaging) }\end{array}$ \\
\hline Jung et al. ${ }^{19}$ & Exploiting sparsity of residuals in dynamic MRI & $\begin{array}{l}\text { Dynamic MRI (cardiac } \\
\text { imaging) }\end{array}$ \\
\hline Yun et al. ${ }^{13}$ & $\begin{array}{l}\text { Exploiting a reference image for basis functions generation used to improve } \\
\text { dynamic MRI }\end{array}$ & Dynamic MRI (brain fMRI) \\
\hline Samsonov et al. ${ }^{33}$ & $\begin{array}{l}\text { Exploiting sparsity of gradient of difference between baseline and follow-up } \\
\text { scans }\end{array}$ & Longitudinal MRI \\
\hline Chen et al. ${ }^{20}$ & $\begin{array}{l}\text { Exploring the exploitation of a reference frame in } \mathrm{x}-\mathrm{t} \text { and } \mathrm{x}-\mathrm{f} \text { domains in } \\
\text { dynamic MRI }\end{array}$ & $\begin{array}{l}\text { Dynamic MRI (cardiac } \\
\text { imaging) }\end{array}$ \\
\hline Wu et al. ${ }^{24}$ & Using noisy reconstruction as a reference for sorting in parallel imaging & Single-contrast MRI \\
\hline Peng et al. ${ }^{25}$ & Exploiting reference image for sparsifying transform generation & Single-contrast MRI \\
\hline Bilgic et al. ${ }^{28}$ & Exploit similarity of spatial derivatives in multi-contrast MRI & Multi-contrast MRI \\
\hline $\begin{array}{l}\text { Du et al. }{ }^{26} \text { and Lam } \\
\text { et al. }{ }^{27}\end{array}$ & $\begin{array}{l}\text { Exploiting similarity to a reference image in a CS-based hybrid } \\
\text { reconstruction and registration scheme }\end{array}$ & Single-contrast MRI \\
\hline Nguyen et al. ${ }^{14}$ & $\begin{array}{l}\text { Exploiting a reference image for generation of basis functions used for } \\
\text { generalized series reconstruction of dynamic MRI }\end{array}$ & Dynamic MRI (brain fMRI) \\
\hline Haldar et al. ${ }^{15}$ & Using structural MRI for SNR improvement of DWI in an ML scheme & Diffusion MRI \\
\hline Qu et al. ${ }^{29,30}$ & $\begin{array}{l}\text { Exploiting similarity of image patches within and between multi-contrast } \\
\text { MRI in CS framework }\end{array}$ & Multi-contrast MRI \\
\hline Huang et al. ${ }^{31}$ & Joint TV and group wavelet based reconstruction for multi-contrast MRI & Multi-contrast MRI \\
\hline Chiew et al. ${ }^{21}$ & Low-rank based reconstruction & Dynamic MRI (brain fMRI) \\
\hline Li et al. ${ }^{34}$ & $\begin{array}{l}\text { Using non reference-based reconstruction as a prior for reference-based } \\
\text { reconstruction }\end{array}$ & Longitudinal MRI \\
\hline Adluru et al. ${ }^{22}$ & $\begin{array}{l}\text { Exploiting TV-based reconstruction for improved low-rank based } \\
\text { reconstruction }\end{array}$ & $\begin{array}{l}\text { Dynamic MRI (cardiac } \\
\text { imaging) }\end{array}$ \\
\hline Otazo et al. ${ }^{23}$ & Low-rank based reconstruction & $\begin{array}{l}\text { Dynamic MRI (cardiac } \\
\text { imaging, MR angiography) }\end{array}$ \\
\hline $\begin{array}{l}\text { Our Method } \\
\text { (FASTMER) }\end{array}$ & Exploiting reference image in an adaptive-weighted CS scheme & $\begin{array}{l}\text { Single- and Multi-contrast } \\
\text { MRI, Longitudinal MRI }\end{array}$ \\
\hline
\end{tabular}

In this paper we present a scheme to exploit a reference image that is applicable for various MRI applications. The major contributions of this paper are: (a) exploiting similarity of intensity values to a reference scan is performed in an adaptive and weighted fashion, taking into account that the reference may exhibit major grey-level differences with respect to the current scan and; (b) several MRI imaging applications are tested (single-contrast high resolution MRI, multi-contrast MRI, and longitudinal MRI), with sampling performed via 
$2 \mathrm{D}$ radial sampling.

More specifically, we introduce a framework, called "FAST MRI by Exploiting a Reference scan" (FASTMER) which is based on two main elements: (a) exploiting both sparsity in the wavelet domain and similarity to a reference scan and (b) weighted reconstruction by adaptive selection of weights during the reconstruction process, taking into account the degree of similarity to the reference image. The adaptive weights in our framework adapt the reconstruction to the actual similarity between the scans. Therefore, it fits a variety of clinical imaging applications with supplemental imaging information, that in many cases is neglected due to its low fidelity.

Experimental results demonstrate the applicability of the proposed method in three different MRI applications that utilize similarity to a reference image. The first application exploits similarity between two different imaging contrasts for fast scanning of one of them. The second example exploits similarity between different scans of the same patient for fast scanning of follow-up scans, and the third application exploits similarity between adjacent slices to improve SNR within the same imaging contrast.

The paper is organized as follows. Section II presents the proposed reference-based MRI approach. Section IV describes experimental results. Section V discusses the method and Section VI concludes by highlighting the key findings of the research.

\section{REFERENCE-BASED MRI}

\section{II.A. Compressed Sensing MRI}

The application of CS for MRI ${ }^{5}$ exploits the fact that MRI scans are typically sparse in a transform domain, which is incoherent with the sampling domain. Nonlinear reconstruction is then used to enforce both sparsity of the image representation in some transform domain and consistency with the acquired data. A typical formulation of CS MRI recovery aims to solve the following unconstrained optimization problem (in a so-called Lagrangian form):

$$
\min _{\mathbf{x}}\left\|\mathbf{F}_{u} \mathbf{x}-\mathbf{y}\right\|_{2}^{2}+\lambda\left\|\Psi_{\mathbf{x}}\right\|_{1}
$$

where $\mathbf{x} \in \mathbb{C}^{N}$ is the $N$-pixel complex image to be reconstructed, represented as a vector, $\mathbf{y} \in \mathbb{C}^{M}$ denotes the k-space measurements, $\mathbf{F}_{u}$ is the undersampled Fourier transform 
operator, $\boldsymbol{\Psi}$ is a sparsifying transform operator and $\lambda$ is a properly chosen regularization parameter. We focus on brain MRI, known to be sparse in the wavelet domain. Therefore, we will assume throughout that $\boldsymbol{\Psi}$ is an appropriately chosen wavelet transform.

This fundamental CS MRI formulation is the basis for many MRI reconstruction applications, where the sparse transform domain varies depending on the particular setting ${ }^{16-19}$. We note that this basic formulation does not take into account any image-based prior information, that exists in many MRI applications.

\section{II.B. Reference-based compressed sensing MRI}

In many MRI imaging scenarios, an a-priori image that may exhibit similarity to the acquired image, is available. This image is coined hereinafter the "reference image" is represented as $\mathbf{x}_{0}$. A reference image could be a different imaging contrast in the same scan, an adjacent image slice or a previous scan of the same patient.

In some imaging applications, we can assume that $\mathbf{x}_{0}$ and $\mathbf{x}$ are similar in most image regions ${ }^{18}$. Therefore the difference $\mathbf{x}-\mathbf{x}_{0}$ can be modelled as sparse, and a CS based optimization may utilize the reference image for improved reconstruction, via $\ell_{1}$ minimization. Such reference-based CS takes into account the fidelity of the measurements and the similarity to the reference scan, as follows:

$$
\min _{\mathbf{x}}\left\|\mathbf{F}_{u} \mathbf{x}-\mathbf{y}\right\|_{2}^{2}+\lambda\left\|\mathbf{x}-\mathbf{x}_{0}\right\|_{1}
$$

This optimization problem assumes high degree of similarity between $\mathbf{x}_{0}$ and $\mathbf{x}$, and is therefore suitable for some specific MRI applications, such as dynamic MRI. However, many MRI applications do not utilize available reference imaging information (for instance, by using (2)) due to the fact that the similarity to the acquired image is partial, not guaranteed or unknown.

We introduce a framework for reference based MRI, which takes into account the fact that $\mathbf{x}_{0}$ may exhibit differences versus $\mathbf{x}$. We also account for the fact that the vector $\mathbf{y}$ may represent multiple images that are contaminated with noise at different levels; we want to prioritize images with low noise standard deviation over ones with high noise standard devi-

ation in the reconstruction process. Our approach is based on enforcing similarity between 
$\mathbf{x}$ and $\mathbf{x}_{0}$ via a weighted $\ell_{1}$ norm:

$$
\min _{\mathbf{x}}\left\|\mathbf{A}\left(\mathbf{F}_{u} \mathbf{x}-\mathbf{y}\right)\right\|_{2}^{2}+\lambda_{1}\left\|\mathbf{W}_{1} \mathbf{\Psi} \mathbf{x}\right\|_{1}+\lambda_{2}\left\|\mathbf{W}_{2}\left(\mathbf{x}-\mathbf{x}_{0}\right)\right\|_{1}
$$

where $\mathbf{A}$ is a diagonal matrix that controls the weight given to the fidelity of certain measurements (used to prioritize samples taken from images with low noise standard deviation). The

matrices $\mathbf{W}_{1}$ and $\mathbf{W}_{2}$ are weighting matrices, $\mathbf{W}_{k}=\operatorname{diag}\left(\left[w_{k}^{1}, w_{k}^{2}, \ldots, w_{k}^{N}\right]\right)$ with $0 \leq w_{k}^{i} \leq 1$, that control the weight given to each element in the sparse representation. In particular, $\mathbf{W}_{1}$ is used to weight specific wavelet atoms in the reconstruction process and $\mathbf{W}_{2}$ is used to weight image regions according to their similarity level with the reference scan. The parameters $\lambda_{1}$ and $\lambda_{2}$ are regularization parameters that control the weight given to each term in the optimization problem.

In most cases, the expected noise level of the acquired data is known and the matrix $\mathbf{A}$ can be determined in advance. As to $\mathbf{W}_{1}$ and $\mathbf{W}_{2}$, there are cases in which neither the similarity to the reference image nor the support in the wavelet domain are known in advance, and therefore these weighting matrices have to be determined during the acquisition process as we describe in the next section.

\section{II.C. Adaptive weighting for reference based MRI}

Since the similarity of $\mathbf{x}$ to $\mathbf{x}_{0}$, as well as the support of $\mathbf{x}$ in the wavelet domain, are unknown, we estimate the matrices $\mathbf{W}_{1}$ and $\mathbf{W}_{2}$ from the acquired data, in an adaptive fashion. Inspired by Weighted-CS ${ }^{47}$, we propose an iterative reconstruction algorithm, where in each iteration a few k-space samples are added to the reconstruction process, based on their distance from the origin of the k-space (samples closer to the origin of the k-space are added first). The rationale lies in the the structure of the minimization problem (3). Due to the fact that (3) is a non-convex minimization problem, we would like to avoid convergence to local minima. Therefore, we experimentally experienced that when we gradually add $\mathrm{k}$-space samples at each iteration the convergence process is more dynamic and improved results are obtained. At the end of each iteration, $\hat{\mathbf{x}}$ is estimated, to serve as the basis for estimating the weighting matrices in the next iteration.

Our rationale behind the iterative computation of $\mathbf{W}_{k}$ is as follows. For $\mathbf{W}_{1}$, we would 
like to relax the demand for sparsity on elements in the support of $\boldsymbol{\Psi}_{\mathbf{x}}$. For $\mathbf{W}_{2}$, we would like to enforce sparsity only in spatial regions where $\mathbf{x} \approx \mathbf{x}_{0}$. We note that when one of the weights is given a small value, for instance, $w_{2}^{i} \rightarrow 0$, then the sparsity on the corresponding image pixel, $\mathbf{x}_{i}$, is relaxed, and vice-versa; when $w_{2}^{i} \rightarrow 1$, sparsity is enforced on $\mathbf{x}_{i}$ (i.e. the $\ell_{1}$ minimization would prefer solutions where $\mathbf{x}_{i} \rightarrow 0$ ).

Since $\mathbf{x}$ is unknown, $\hat{\mathbf{x}}$, updated in every iteration, is used instead. The elements of the weighting matrices are then chosen as follows:

$$
\begin{aligned}
w_{1}^{i} & =\frac{1}{1+[|\boldsymbol{\Psi} \hat{\mathbf{x}}|]_{i}} \\
w_{2}^{i} & =\frac{1}{1+\left[\left|\hat{\mathbf{x}}-\mathbf{x}_{0}\right|\right]_{i}}
\end{aligned}
$$

where $[\cdot]_{i}$ denotes the $i$ th element of the vector in brackets. We note that in a similar way to Candès' approach ${ }^{47}$, developed for $\mathbf{x}$ which is truly sparse, the weights in (4) are given values that vary between 0 and 1 . The values for $w_{1}^{i}$ and $w_{2}^{i}$ are inversely proportional to those of the corresponding elements in the vectors $\Psi_{\mathbf{X}}$ and $\hat{\mathbf{x}}-\hat{\mathbf{x}}_{0}$, respectively. Therefore, since reconstruction quality improves at each consecutive iteration, we get $w_{2}^{i} \rightarrow 1$ in regions where $\mathbf{x} \sim \mathbf{x}_{0}$, thereby enforcing sparsity of the difference $\mathbf{x}-\mathbf{x}_{0}$ in those regions. The same analysis applies for $\mathbf{W}_{1}$ and the representation of the image in the wavelet domain.

The proposed algorithm is coined FAST MRI by Exploiting a Reference scan (FASTMER) and is summarized in Algorithm 1. Note that in the first iteration of the algorithm we do not assume similarity with the reference image (i.e., we set $\mathbf{W}_{1}=\mathbf{I}$ and $\mathbf{W}_{2}=\mathbf{0}$ for the first iteration). This is done in order to prevent the algorithm from convergence to an incorrect solution in cases where similarity between scans does not exist. 


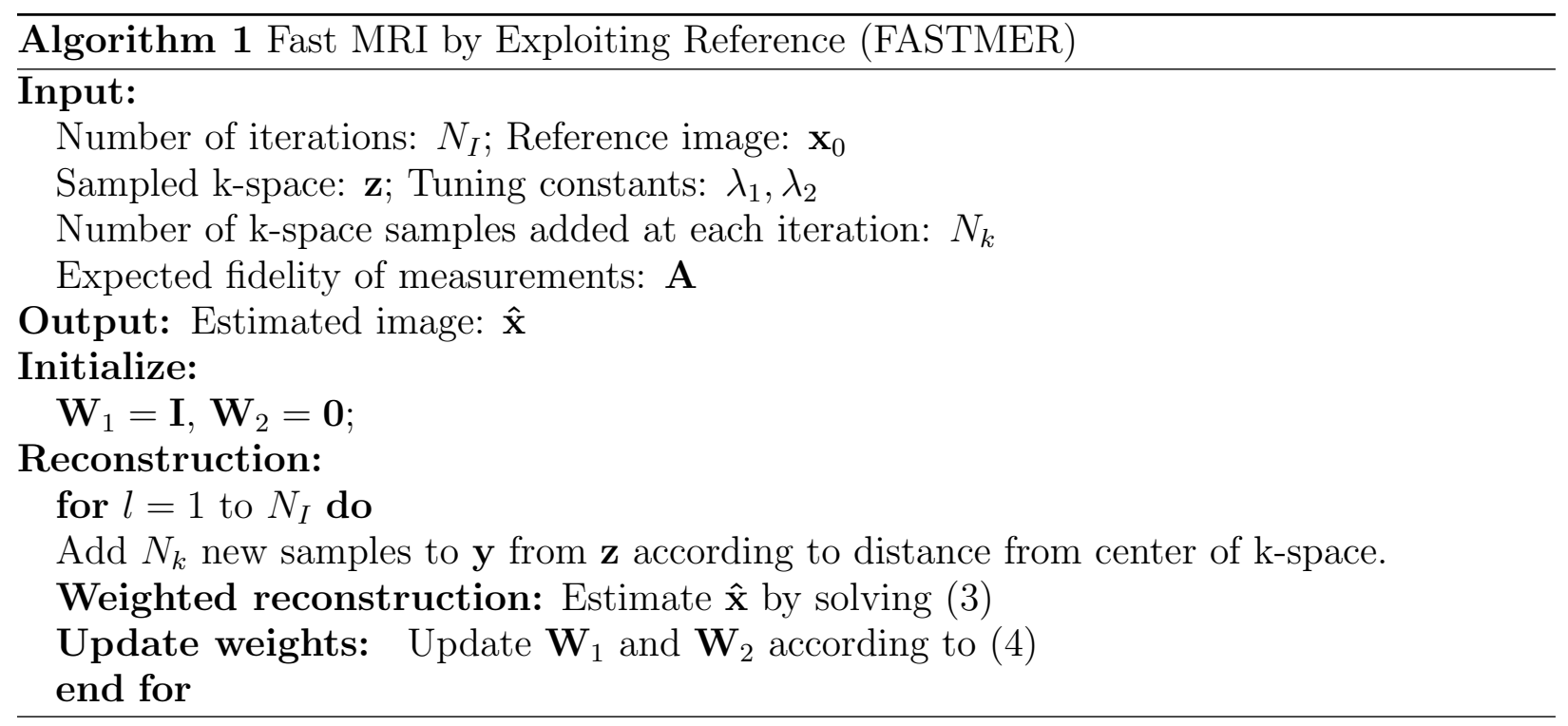

To solve the $\ell_{1}$-minimization problem (3) in the weighted reconstruction phase, we use an extension of SFISTA ${ }^{48}$. The extended algorithm is summarized in Algorithm 2, where the notation $\|\cdot\|_{2}$ for matrices denotes the largest singular value. The operator $\Gamma_{\lambda \mu}(\mathbf{z})$ is the soft shrinkage operator, which operates element-wise on $\mathbf{z}$ and is defined as (for complex valued $\left.z_{i}\right)$ :

$$
\Gamma_{\lambda \mu}\left(z_{i}\right)= \begin{cases}\frac{\left|z_{i}\right|-\lambda \mu}{\left|z_{i}\right|} z_{i}, & \left|z_{i}\right|>\lambda \mu \\ 0, & \text { otherwise }\end{cases}
$$




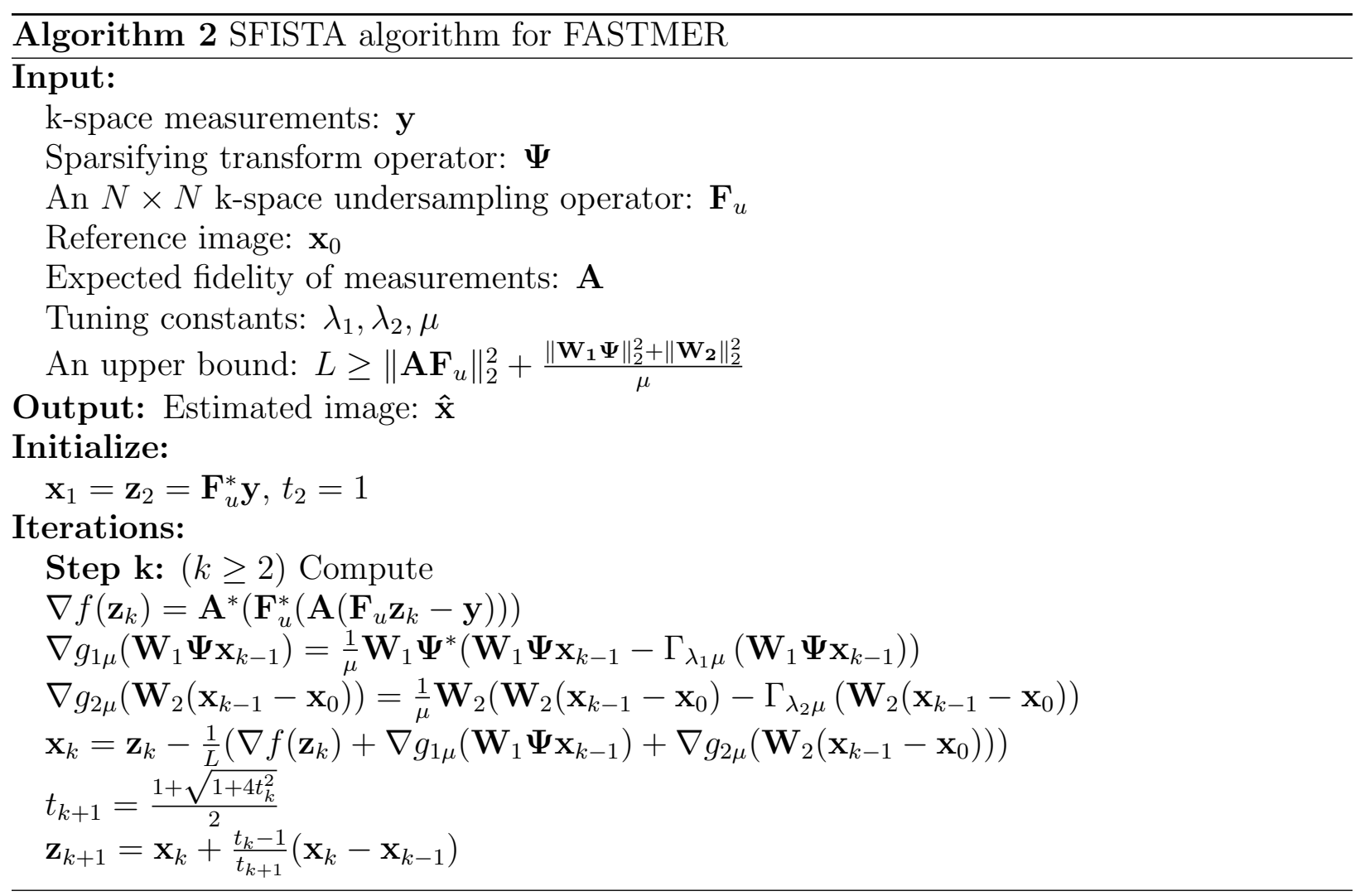

Algorithm 2 minimizes (3), where the trade-off between the two sparsity assumptions is controlled by the ratio between $\lambda_{1}$ and $\lambda_{2}$, via $\Gamma(\cdot)$, and the overall convergence is controlled by $\mu$.

\section{FASTMER FOR SNR IMPROVEMENT}

In MRI, SNR is proportional to the number of protons involved in generating the measured signal. As a result, thick slices provide better SNR than thin ones. However, the thinner the slice, the better the image resolution in the z-axis. Therefore, to obtain high quality MRI for clinical evaluation purposes, high SNR MRI that consists of thin slices is required. The common approach today for SNR improvement of MRI with thin slices consists of averaging over several excitations (usually three or four), which extends the scanning time by the same amount.

In this application, where thin slices are acquired, one may consider shortening scanning time by reducing the number of excitations and exploiting similarity between thin slices. In addition, similarity to a thick, high SNR image slice that overlaps the thin slices can also 
be utilized for SNR improvement.

We will consider a specific implementation, where a single excitation is used to acquire two thin adjacent slices with low SNR, $\mathbf{x}_{1}$ and $\mathbf{x}_{2}$, and a single thick slice, $\mathbf{x}_{3}$ that spatially overlaps $\mathbf{x}_{1}$ and $\mathbf{x}_{2}$. Obviously, our goal is to improve the SNR of $\mathbf{x}_{1}$ and $\mathbf{x}_{2}$ taking into account the similarity between them and the high-SNR, thick slice, $\mathbf{x}_{3}$. In this specific example we perform a total of three acquisitions for high SNR reconstruction of two adjacent slices, instead of 8 acquisitions (4 excitations for each thin slice) in the conventional, multiple excitations-based approach.

To introduce the method within our framework, let

$$
\mathbf{y}=\left[\begin{array}{l}
\mathbf{y}_{1} \\
\mathbf{y}_{2} \\
\mathbf{y}_{3}
\end{array}\right], \quad \mathbf{x}=\left[\begin{array}{c}
\mathbf{x}_{1} \\
\mathbf{x}_{2} \\
0.5\left(\mathbf{x}_{1}+\mathbf{x}_{2}\right)
\end{array}\right]
$$

where $\mathbf{y}$ represents the k-spaces of two thin slices and the corresponding thick one, respectively, and $\mathbf{x}$ represents the two thin slices and their average, which corresponds to the overlapping thin slice. The matrix $\mathbf{A}$ is determined by the estimated noise level of the elements in $\mathbf{y}$, such that $\mathbf{A}=\operatorname{diag}\left(\frac{1}{\sigma_{1}} \mathbf{I}_{N}, \frac{1}{\sigma_{2}} \mathbf{I}_{N}, \frac{1}{\sigma_{2}} \mathbf{I}_{N}\right)$ where $\left\{\sigma_{i}\right\}_{i=1}^{3}$ are the noise standard deviation of $\left\{\mathbf{y}_{i}\right\}_{i=1}^{3}$, respectively and $\mathbf{I}_{N}$ is an identity matrix of size $N$. Similarity is enforced between the thin slices, and (3) is reformulated as:

$$
\min _{\mathbf{x}}\left\|\mathbf{A}\left(\mathbf{F}_{3} \mathbf{x}-\mathbf{y}\right)\right\|_{2}^{2}+\lambda_{1}\left\|\mathbf{W}_{1} \mathbf{\Psi}_{3} \mathbf{x}\right\|_{1}+\lambda_{2}\left\|\mathbf{W}_{2} \mathbf{B} \mathbf{x}\right\|_{1}
$$

Here $\mathbf{F}_{3}=\operatorname{diag}([\mathbf{F}, \mathbf{F}, \mathbf{F}])$ is a block diagonal matrix, with three Fourier matrices on the main diagonal, $\boldsymbol{\Psi}_{3}=\operatorname{diag}([\boldsymbol{\Psi}, \boldsymbol{\Psi}, \boldsymbol{\Psi}])$ and $\mathbf{B}=\left[\begin{array}{lll}\mathbf{I}_{N} & -\mathbf{I}_{N} & \mathbf{0}\end{array}\right]$.

Similarity between the thick slice to the average between the thin slices is enforced in the Fourier domain, via the leftmost term of (7). Adapting Algorithm 1 and Algorithm 2 to solve (7) is straightforward, the final method appears in Appendix A.

\section{EXPERIMENTAL RESULTS}

To demonstrate the performance of our reference based MRI approach we examine three MRI applications, all of which utilize a reference scan for improved reconstruction. Where 
relevant, partial k-space acquisition was obtained by 2D radial sampling of a fully sampled k-space. The angles of the trajectories were taken randomly according to a uniform distribution. Non-uniform sampling and reconstruction was performed using the non-uniform Fourier transform (NUFFT) package of Fessler et al. ${ }^{49}$. A Daubechies-4 wavelet transform was used as the sparsifying transform. Different values of $\lambda_{1}, \lambda_{2}$ in the range of [0,0.9] were examined, and the best result in terms of image quality is presented in each case (see Section IV.D). We used $\mu=10^{-3}\left(\frac{\lambda_{1}+\lambda_{2}}{2}\right)^{-1}$ in our experiments. The number of iterations used in Algorithm 2 for the results obtained in this section is between 30 and 50 iterations.

All scans were performed on a GE Signa 1.5T HDx scanner, using a 8-channel head coil and with matrix size of $320 \times 320$ and FOV of $20 \mathrm{~cm}$ for each in-plane direction. High SNR images reconstructed from fully sampled, multiple excitations data serve as the gold standard. The source code and data required to reproduce the results presented in the this paper can be downloaded from: http://www.technion.ac.il/ weizmanl/software

To provide a quantitative measure for the results, we examine the peak signal-to-noise ratio (PSNR) of each experiment, defined as: $\mathrm{PSNR}=10 \log _{10}\left(M^{2} / V_{s}\right)$, where $M$ denotes the maximum possible pixel value in the image and $V_{s}$ is the Mean Squared Error (MSE) between the original image, $\mathbf{x}$ and the reconstructed image, $\hat{\mathbf{x}}$. The resulting weighting matrices $\left(\mathbf{W}_{1}\right.$ and $\mathbf{W}_{2}$ ) are presented in Appendix $\mathrm{B}$, as well as the similarity maps between the reference image and the reconstructed image.

Since the proposed approach is based on the difference between the reference image from the reconstruction, we need to verify that both images are aligned and have matched intensities. In the experiments presented hereinafter we conducted a wavelet-based reconstruction

first (according to (1)) using the acquired data, to get a rough estimate on the alignment parameters and the grey-level range of the reconstructed image. Then, we embedded the extracted parameters in our reconstruction process for successful reference-based reconstruction. This issue is further discussed in Section V.C.

\section{IV.A. Utilizing similarity between T2-weighted and FLAIR}

In this experiment, our goal is to reconstruct a FLAIR image, $\mathbf{x}$, from undersampled measurements, utilizing similarity to a T2-weighted image. Images were acquired with slice thickness of $4 \mathrm{~mm}$. FLAIR acquisitions parameters were: TE $=123 \mathrm{~ms}$, TR=8000 $\mathrm{ms}$ and 


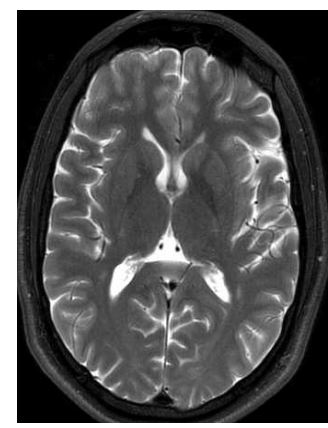

T2-weighted

FASTMER result

(gold standard)

$(\mathrm{PSNR}=33 \mathrm{~dB})$

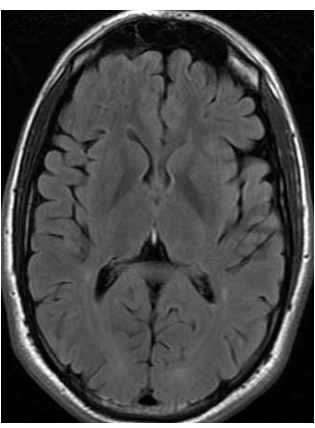

FLAIR

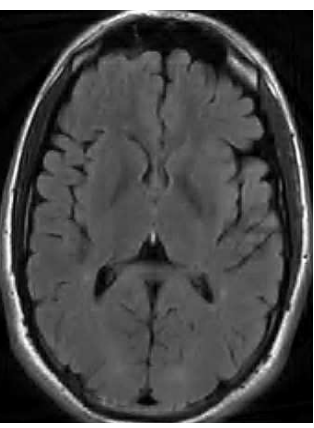

Wavelet+TV result

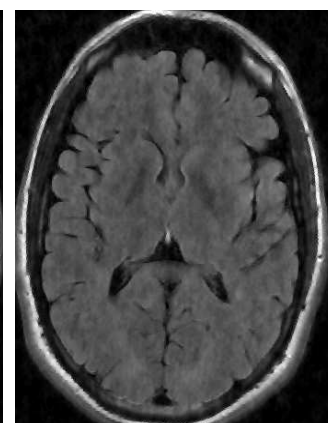

Bilgic et al. ${ }^{28}$ result

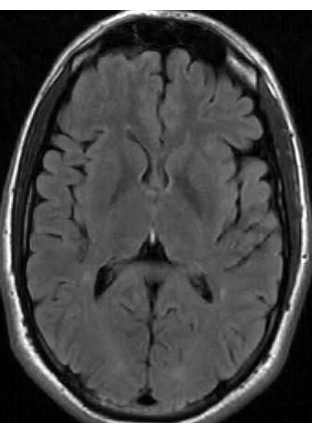

$(\mathrm{PSNR}=30 \mathrm{~dB})$

FIG. 1 FASTMER used within the same scan: reconstruction results utilizing similarity between $\mathrm{T} 2$ and FLAIR contrasts. The two leftmost images are the T2 and FLAIR images reconstructed from $100 \%$ of $\mathrm{k}$-space data. The three rightmost images show reconstruction results from $25 \%$ of k-space FLAIR data, for wavelet+TV based reconstruction, the method of Bilgic et al. for multi-contrast reconstruction using Bayesian- $\mathrm{CS}^{28}$ and our proposed, reference-based approach (FASTMER). The numbers in brackets are the PSNR values vs. the FLAIR gold standard.

$\mathrm{TI}=2000 \mathrm{~ms}$, and T2-weighted acquisition parameters were: $\mathrm{TE}=68.4 \mathrm{~ms}$ and $\mathrm{TR}=6880 \mathrm{~ms}$. We sampled only $25 \%$ of the FLAIR k-space with radial sampling and utilized the fully sampled T2-weighted scan as the reference image $\mathbf{x}_{0}$. Since all samples were acquired with similar noise level, $\mathbf{A}=\mathbf{I}$.

To provide a baseline for comparison, we compared FASTMER to two methods. The first is reconstruction based on sparsity in the wavelet domain and enforces total-variation $(\text { wavelet }+\mathrm{TV})^{5}$ using undersampled FLAIR data only. The second is the algorithm of Bilgic et al. ${ }^{28}$ which exploits the similarity of gradients between different contrasts.

Figure 1 shows the fully sampled T2 and FLAIR images, the wavelet+TV based reconstruction, the reconstruction using the method of Bilgic et al. ${ }^{28}$ and the result of FASTMER. In addition, the PSNR values of each reconstruction method vs. the gold-standard are provided. It can clearly be seen that FLAIR reconstruction with FASTMER outperforms the two other methods used for comparison, using only $25 \%$ of the data. 


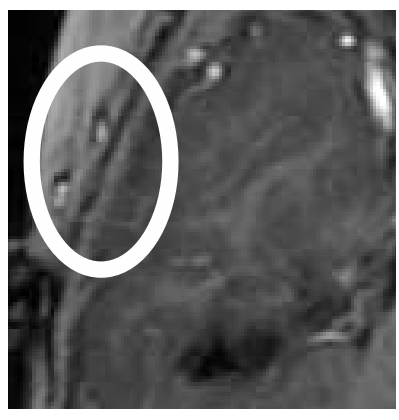

Gold standard (zoom)

FASTMER (zoom)

$(\mathrm{PSNR}=37 \mathrm{~dB})$

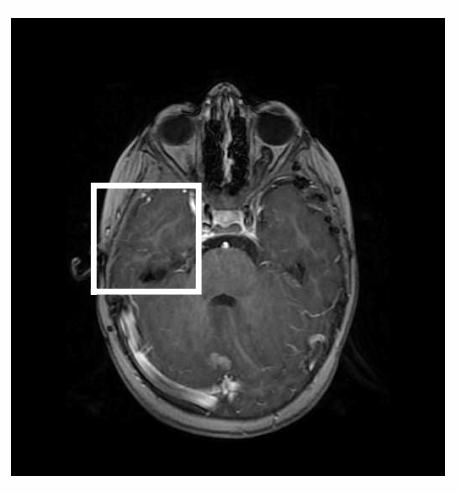

Gold standard
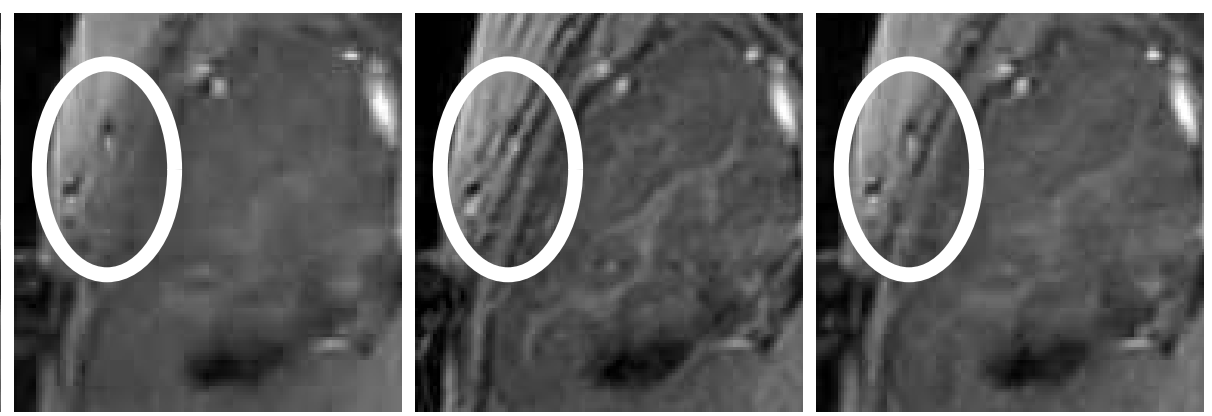

Wavelet+TV recon. (zoom)

Samsonov et al. (zoom)

$$
(\mathrm{PSNR}=34 \mathrm{~dB}) \quad(\mathrm{PSNR}=35 \mathrm{~dB})
$$

FIG. 2 FASTMER used in longitudinal studies: reconstruction results from $25 \%$ of k-space data. Bottom left: ground truth followup image reconstructed from $100 \%$ of $\mathrm{k}$-space data. The rectangle defines the region-of-interest explored in this figure. Upper row: Enlarged versions of the region-of-interest in the ground truth image (leftmost image), followed by the results of wavelet+TV based reconstruction, the method of Samsonov et al. for longitudinal studies ${ }^{33}$ and our proposed, reference-based approach (FASTMER). The numbers in brackets are the PSNR values vs. the gold standard. It can be seen that FASTMER exhibits results which are very similar to the gold standard, and reveals imaging features that are blurred or not visible in other recoveries.

\section{IV.B. Utilizing similarity between baseline and follow-up scans}

Repeated brain MRI scans of the same patient every few weeks or months are very common for follow-up of brain tumors. Here, our goal is to use a previous scan in the time series as a reference scan for reconstruction of a follow-up scan. In this application we need to take into account that similarity between the reference and current scans is not guaranteed (e.g. due to pathology changes), and prior information on spatial regions that may exhibit differences is not available. These obstacles are discussed thoroughly in our previous publication ${ }^{40}$ and in Section V.C and Appendix C of this paper. Since all samples are acquired with similar noise level, we set $\mathbf{A}=\mathbf{I}$.

We compared FASTMER to two methods. The first is wavelet+TV based reconstruction. The second is the method of Samsonov et al. ${ }^{33}$ which exploits the gradient images similarity between a follow-up scan and a baseline scan using Bayesian-CS, in a non-weighted approach. 
Figure 2 shows reconstruction results of a follow-up contrast enhanced T1-weighted brain scan utilizing the baseline scan as reference $(\mathrm{TE}=11.5 \mathrm{~ms}, \mathrm{TR}=520 \mathrm{~ms}$ slice thickness: $1 \mathrm{~mm}$ for both scans). Results were obtained using only $25 \%$ of $\mathrm{k}$-space data. It can be seen that FASTMER exhibits imaging features that are hardly visible in both of the methods it is compared against. In addition, the PSNR values of each reconstruction algorithm vs. the gold-standard are provided. The superiority of our approach is achieved thanks to the iterative mechanism that adapts the reconstruction to match actual similarity.

\section{IV.C. Utilizing similarity between adjacent slices}

In this application we examine the extension of fast reference based MRI detailed in Section III to improve SNR of thin MRI slices. We acquired a brain T2-weighted scan with slice thickness of $0.8 \mathrm{~mm}$ followed by an additional acquisition with slice thickness of $1.6 \mathrm{~mm}$ ( $\mathrm{TE}=68.4 \mathrm{~ms}$ and $\mathrm{TR}=6880 \mathrm{~ms}$ for all scans). In all scans a single excitation was used. As a result, we obtained a low SNR scan consisting of thin slices, and high SNR scan consisting of thick slices where each thick slice overlaps two thin ones. Our goal is to reconstruct a high SNR scan comprised of thin slices from this data.

Figure 3 shows thin slices acquired using 4 excitations (NEX=4), used as our gold standard in this experiment, and the noisy input images that were acquired with a single excitation $(\mathrm{NEX}=1)$. Here, we compare our method to a wavelet+TV based approach, which has been tested previously to improve $\mathrm{SNR}$ in $\mathrm{MRI}^{6}$.

In terms of scanning time, 4 excitations are required to obtain thin slices with SNR comparable to SNR of data reconstructed with FASTMER. Therefore, without additional acceleration techniques (parallel imaging etc.), our approach requires scanning 3 slices once versus scanning 2 slices 4 times in conventional scanning, yielding a speed-up factor of 2.6.

\section{IV.D. Parameter sensitivity analysis}

In this analysis we examine the sensitivity of FASTMER to changes in the regularization parameters of the algorithm. Figure 4 shows the PSNR results as $\lambda_{1}$ and $\lambda_{2}$ vary. The values shown for the adjacent slices experiment were averaged over the two thin slices used in the experiment. Generally, we observe that lower values of $\lambda_{1}$ and $\lambda_{2}$, with $\lambda_{1}<\lambda_{2}$ provide 

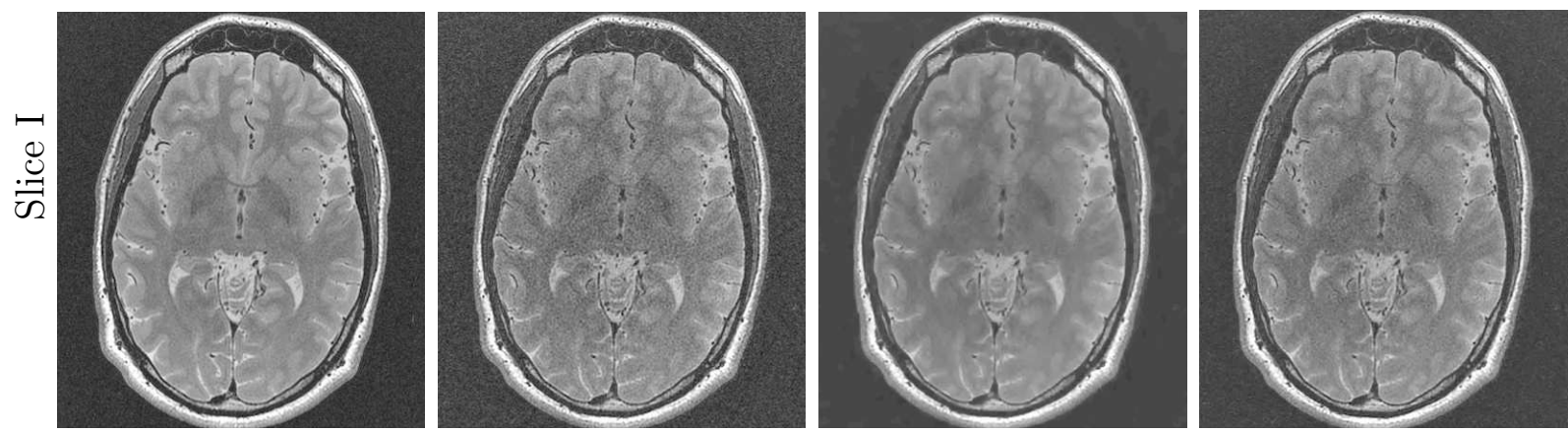

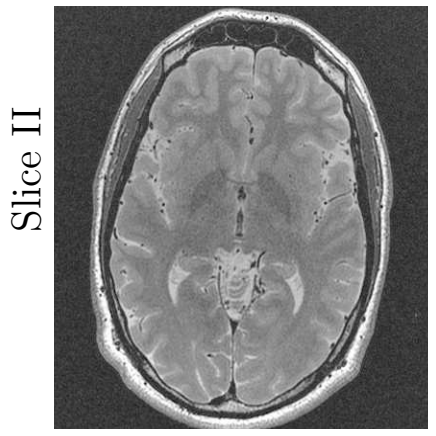

Gold standard

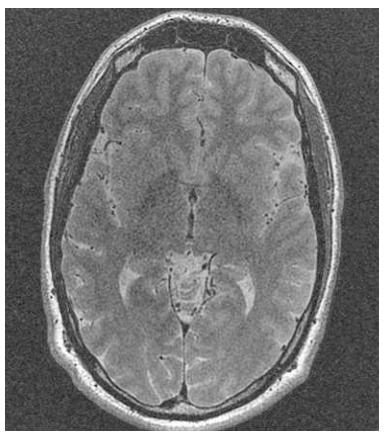

Original noisy images
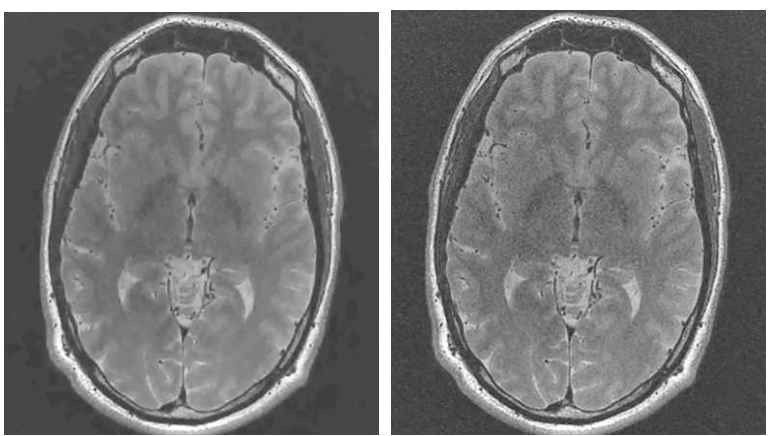

Wavelet+TV result

FASTMER result
$(\mathrm{NEX}=1)$
$(\mathrm{NEX}=4)$

$(\mathrm{PSNR}=34 \mathrm{~dB})$

FIG. 3 FASTMER used within the same imaging contrast: reconstruction results from low SNR data. Each row corresponds to a single slice, from two adjacent slices. It can be seen that high similarity exists within adjacent slices, which can be exploited to improve SNR. The left most column shows the gold standard, acquired using four excitations (NEX=4). The input to our approach is presented as the original noisy images, acquired with single acquisition $(\mathrm{NEX}=1)$, followed by the result of a Total-Variation (TV)-based reconstruction. The rightmost column shows FASTMER recovery. The numbers in brackets are the PSNR values vs. the gold standard (values were averaged over the two slices used in the experiment).

reasonable PSNR. This can be explained by the fact that over-promoting sparsity versus consistency to measurements degrades the reconstruction quality. In addition, we see that the T2-FLAIR experiment provides a lower range of PSNR values in comparison to other experiments. This can be explained by the fact that similarity is not enforced over the entire image in this case, due to many regions of differences between FLAIR and T2. 

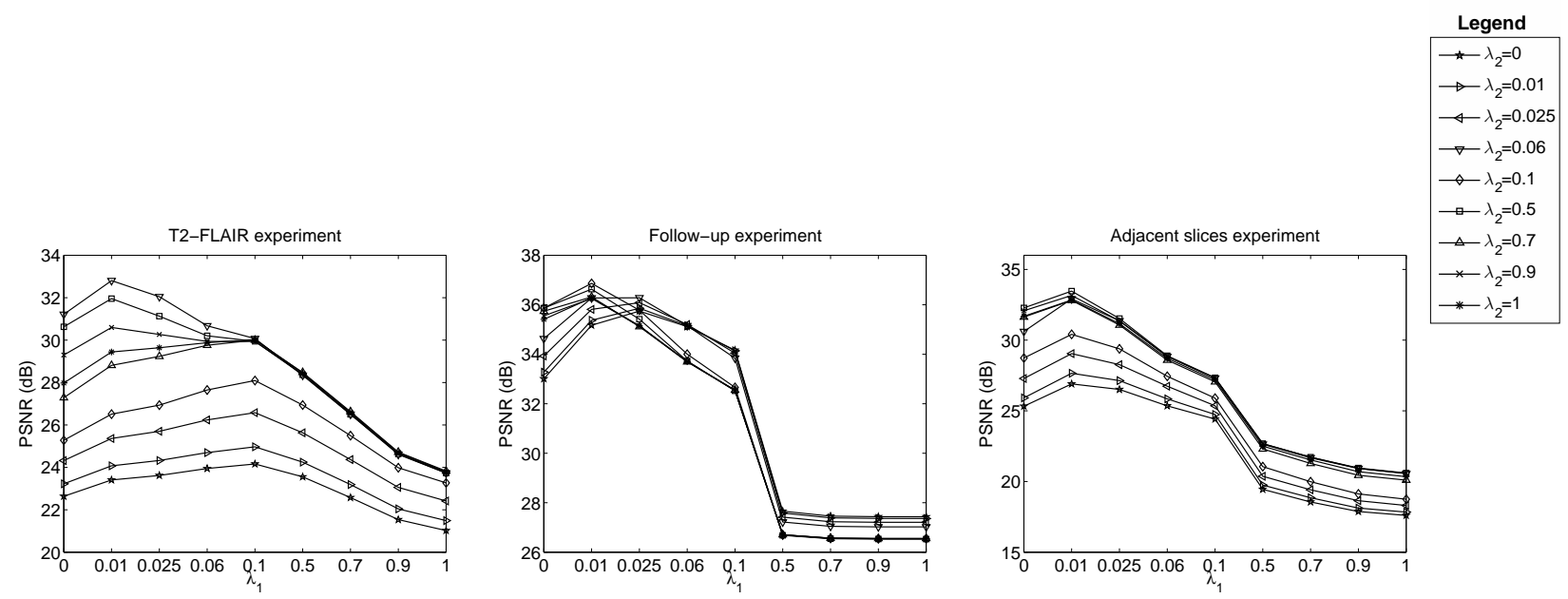

FIG. 4 Sensitivity analysis: PSNR results of T2-FLAIR (left), follow-up (middle) and adjacent slice similarity (top) applications for various values of $\lambda_{1}$ and $\lambda_{2}$. Each line represents a different value of $\lambda_{2}$, according to the legend at the left.

\section{DISCUSSION}

\section{V.A. Theoretical justification}

The algorithm presented in our paper generalizes the reweighted $\ell_{1}$ minimization method by Candes, Wakin, and Boyd ${ }^{47}$ to the case where there is side information (the referenceimage in FASTMER). Their algorithm is quite intuitive and works very well in practice, and their analytical justification can easily be extended to our case. To analyze the theoretical aspects of our weighting algorithm, we explored our model from a signal processing point of view $^{50}$.

We developed a bound on the number of measurements required for perfect reconstruction of $\mathbf{x}$ with high probability for the case where side information exists. We derived a weighted reconstruction scheme based on the bound. We have shown that adding the weights to our scheme highly improves the results in comparison to other non-weighted $\ell_{1}$-minimization based solutions. The results of this work have been published ${ }^{50}$ and the reader is referred to this recent publication to delve into the theoretical aspects of this work.

\section{V.B. Adaptive sampling}

In our approach all data is acquired at once. Several recent publications suggest that prior knowledge can also be used to optimize data acquisition ${ }^{51-53}$. However, since in our 
framework the similarity between scans is not guaranteed, we avoid using prior information during sampling. Another way of utilizing the reference image in the sampling stage would be to acquire a small number of samples in each iteration based on the reconstruction results, in an adaptive manner ${ }^{41,54-57}$. This approach, which requires image reconstruction at each iteration as part of the sampling process, has been tested in our previous work ${ }^{40}$. It was shown to be time consuming leading to substantial increase of scanning time if not programmed in hardware or accelerated by other means.

\section{V.C. Practical limitations}

FASTMER would provide the best results if the reference scan and the acquired scan are spatially aligned and exhibit a similar range of grey-level intensities. While these assumptions are mostly valid within the same imaging contrast (our slice similarity application), they may not be valid for different contrasts or scans acquired at different times.

The solution to both issues can be obtained by grey-level normalization and realigning after acquisition. Since all data is acquired prior to reconstruction, a wavelet based recovery using all samples can be performed first. Although it may exhibit poor reconstruction of fine details (as presented in our experiments), it was found to be sufficient for grey-level normalization and alignment parameter extraction. Then, the extracted parameters are used for normalization and realignment of the data to improve reconstruction performance. An additional approach that is currently left for future research is to examine more complex similarity measures (as opposed to the grey-level subtraction proposed in this paper), e.g. non-linear similarity measures or measures that also take into account misalignement and intensity variation between the images. However, one should take into account that the solution to the corresponding $\ell_{1}$ minimization problem with a non-linear constraint might be more complex than FASTMER

It is worth noting here that if the alignment and normalization processes fail, then our iterative approach will detect the low similarity between the scans. As a result, the reference image will not be taken into account and reconstructed image will converge to a waveletbased reconstruction. This statement is supported by experiments presented in Appendix C. 


\section{CONCLUSIONS}

In this paper we introduced a new framework fast MRI by exploiting a reference image (FASTMER). We developed an iterative reconstruction approach that supports cases in which similarity to the reference scan is not guaranteed. we observe the issue of embedding prior images in MRI reconstruction to accelerate acquisition and improve SNR has been examined in the past, in this paper the similarity to the reference image is learned during reconstruction. As a result, non-valid data is ignored in the reconstruction process, which enables the applicability of the method to a variety of MRI applications.

We demonstrate the performance of our framework in three clinical MRI applications: Reconstruction of noisy, single-contrast data, multi-contrast reconstruction and longitudinal reconstruction. Results exhibit significant improvement versus wavelet+TV based reconstruction and other MRI application-specific approaches.

Thanks to the existence of reference images in various clinical imaging scenarios, the proposed framework can play a major part in improving reconstruction in many MR applications. Future work will consist of applying the method to a wider range of medical imaging settings, such as low dose CT and fMRI, as well as exploring the combination of CS for parallel imaging and the proposed approach.

\section{ACKNOWLEDGEMENTS}

The authors wish to thank the Gilbert Israeli Neurofibromatosis Center (GINFC) for identifying, anonymizing and providing the MRI datasets, and performing the scanning experiments at various NEX values for the application that utilizes similarity between adjacent slices. This work was supported by the Ministry of Science, by the ISF I-CORE joint research center of the Technion and the Weizmann Institute, Israel and by the European Union's Horizon 2020 research and innovation programme under grant agreement No. 646804-ERC-COG-BNYQ.

The authors have no relevant conflicts of interest to disclose. 


\section{Appendix A: Adaptation of Algorithm 1 and 2 for reference-based SNR improvement}

The SNR improvement in Section III requires the solution of (7) in an iterative manner. For this purpose, we define the weights update as follows:

$$
\begin{aligned}
w_{1}^{i} & =\frac{1}{1+\left[\left|\boldsymbol{\Psi}_{3} \hat{\mathbf{x}}\right|\right]_{i}} \\
w_{2}^{i} & =\frac{1}{1+[|\mathbf{B} \hat{\mathbf{x}}|]_{i}} .
\end{aligned}
$$

Below we describe Algorithms 3 and 4, which are adaptations of Algorithms 1 and 2 to this setting, where $\Gamma_{\lambda \mu}(\mathbf{z})$ is defined in $(5), \mathbf{F}_{3}^{*}=\operatorname{diag}\left(\left[\mathbf{F}^{*}, \mathbf{F}^{*}, \mathbf{F}^{*}\right]\right)$ and $\mathbf{\Psi}_{3}^{*}=\operatorname{diag}\left(\left[\boldsymbol{\Psi}^{*}, \mathbf{\Psi}^{*}, \boldsymbol{\Psi}^{*}\right]\right.$.

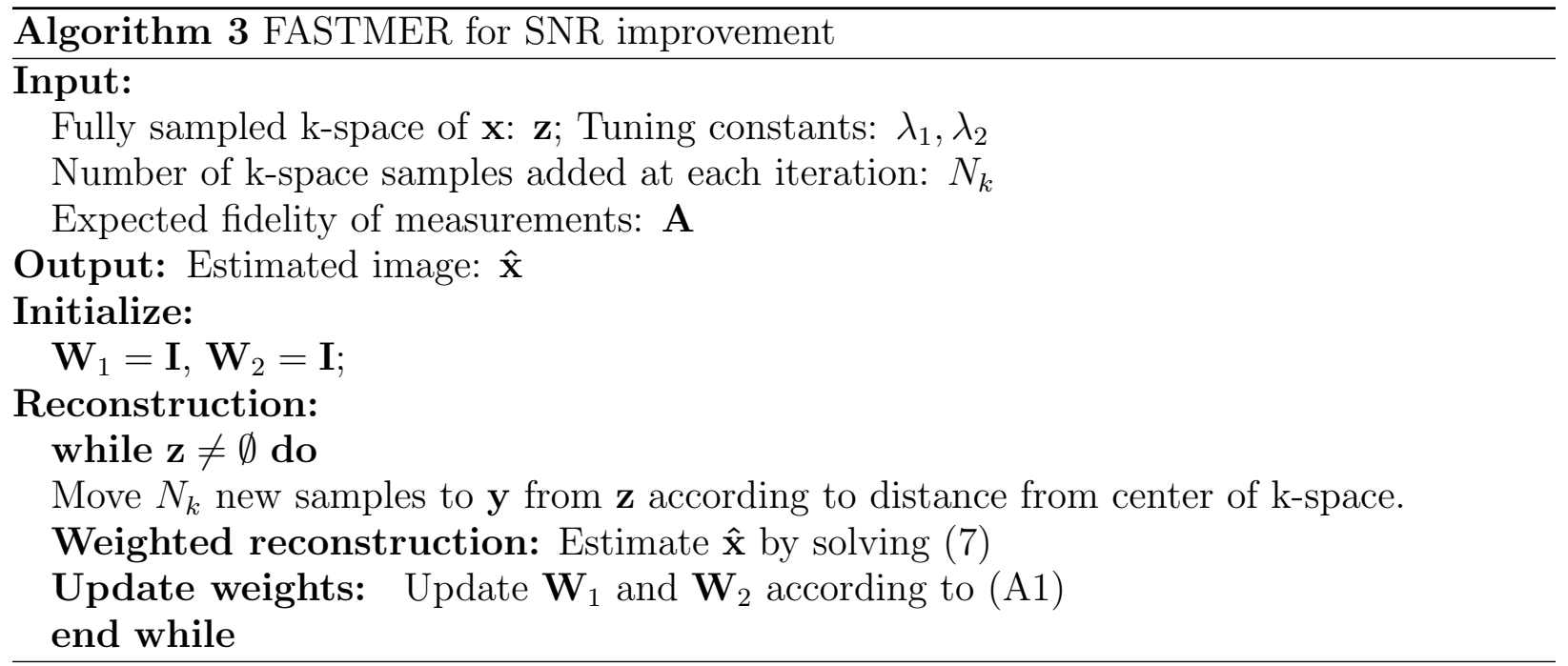




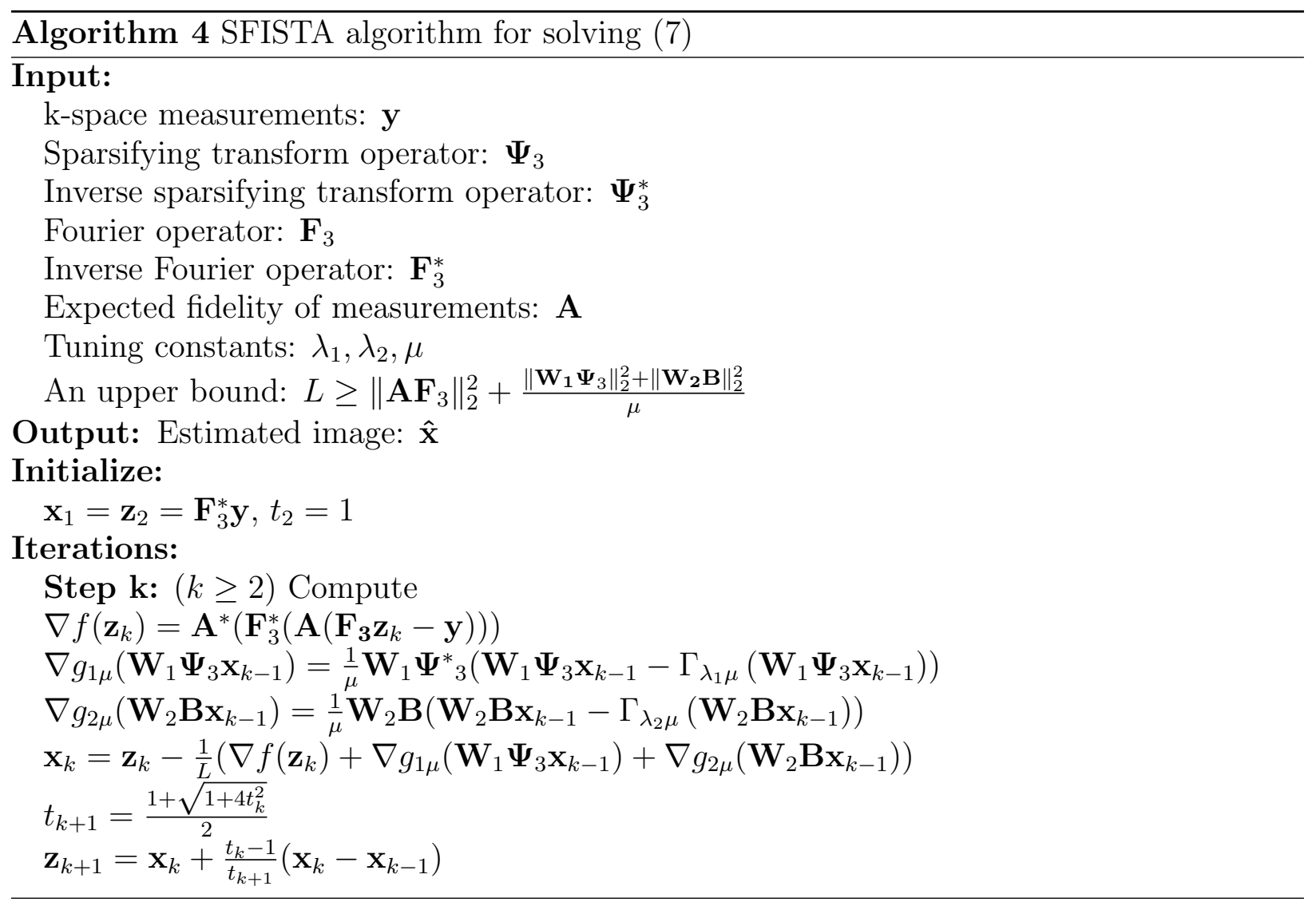

\section{Appendix B: Similarity maps and resulting weighting matrices}

In this appendix we present the similarity maps between each gold-standard to the reference image and the resulting weighting matrices, $\mathbf{W}_{1}$ and $\mathbf{W}_{2}$ for the cases examined in this paper. Note that although in the paper $\mathbf{x}$ is defined as a vector and the weighting matrices are defined as diagonal matrices, $\mathbf{W}_{1}$ and $\mathbf{W}_{2}$ are represented in this section as non-diagonal matrices, used to weight $\mathbf{x}$ represented as a $2 \mathrm{D}$ matrix, for convenience. In addition, the difference images refer to the absolute normalized difference.

Figures 5 and 6 show the maps for the T2-FLAIR and follow-up experiments and Figure 7 shows the maps for the SNR improvement experiment. Here the difference map is computed between the input noisy adjacent slices. 
T2-FLAIR experiment

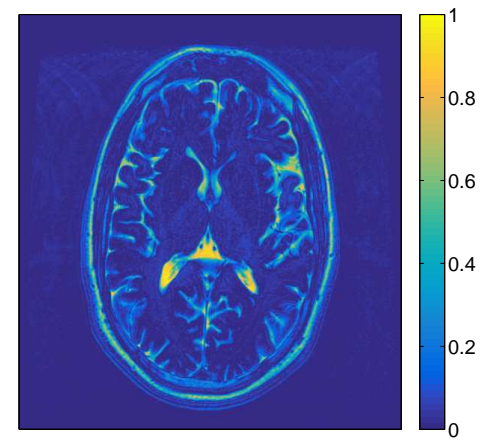

Normalized difference image

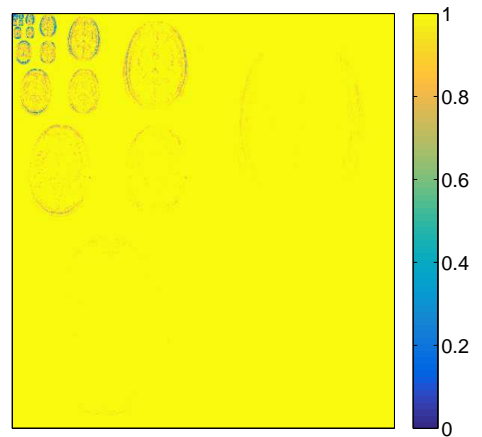

Resulting $\mathbf{W}_{1}$

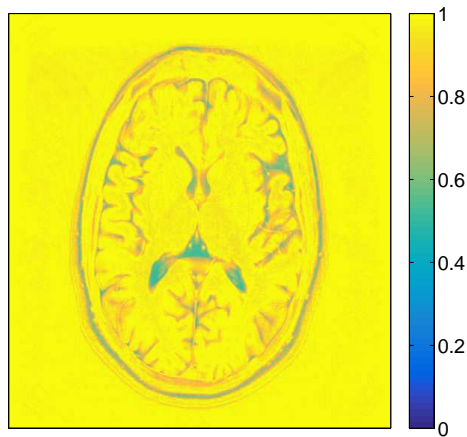

Resulting $\mathbf{W}_{2}$

FIG. 5 T2-FLAIR experiment: Normalized difference image between the gold-standard FLAIR image and the reference T2-weighted image, and the resulting weighting matrices, $\mathbf{W}_{1}$ and $\mathbf{W}_{2}$ of FASTMER.

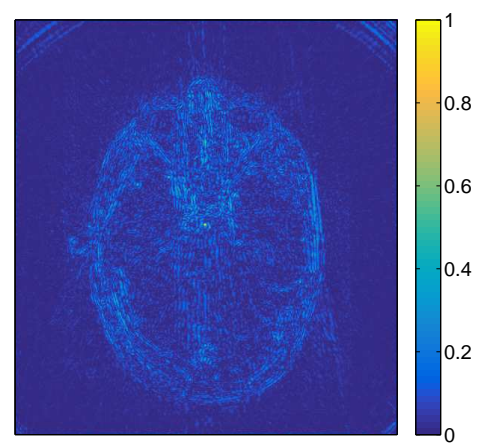

Normalized difference image

Follow-up experiment

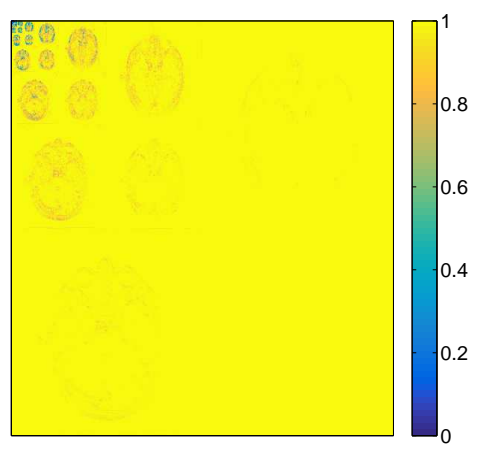

Resulting $\mathbf{W}_{1}$

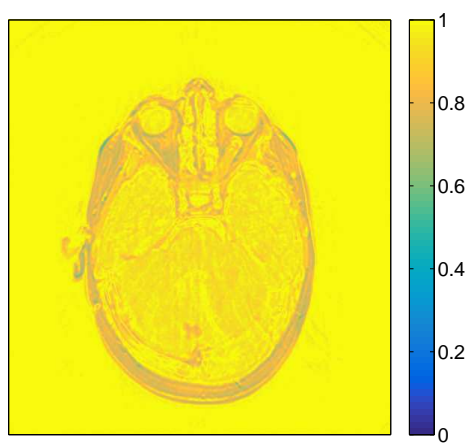

Resulting $\mathbf{W}_{2}$

FIG. 6 Follow up experiment: Normalized difference image between the gold-standard follow-up image and the reference baseline image, and the resulting weighting matrices, $\mathbf{W}_{1}$ and $\mathbf{W}_{2}$ of FASTMER.

\section{Appendix C: Method performance for varying degrees of similarity with the reference image}

To examine the performance of FASTMER for varying degrees of similarity between the reference image and the acquired image we repeated the experiment described in Section IV.A for misregistered reference image. Three scenarios were examined: $5^{\circ}$ intra-plane rotation, $45^{\circ}$ intra-plane rotation, and $5 \mathrm{~mm}$ inter-plane translation together with $5^{\circ}$ rotation. The results are shown in Table II, while the gold-standard, wavelet+TV and FASTMER 
SNR improvement experiment

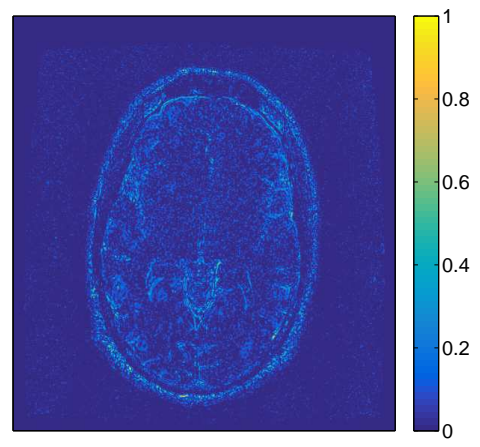

Normalized difference image

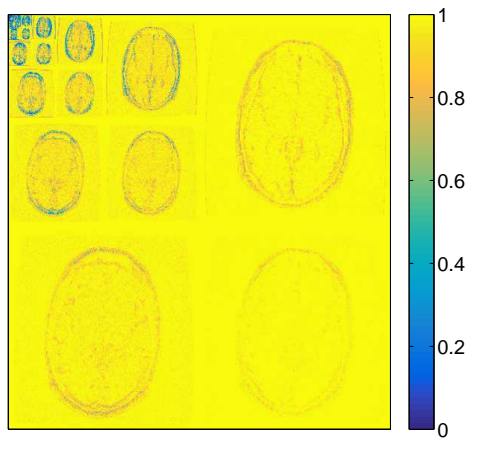

Resulting $\mathbf{W}_{1}$

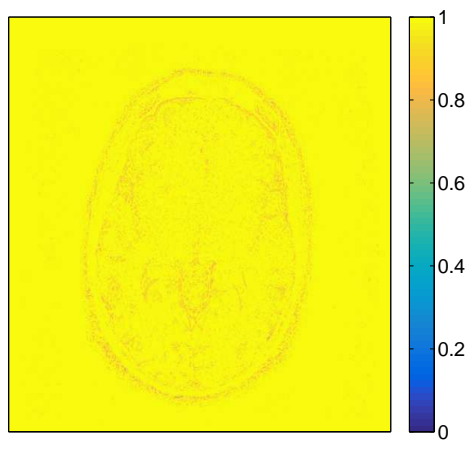

Resulting $\mathbf{W}_{2}$

FIG. 7 SNR improvement experiment: Normalized difference image between the two adjacent, low SNR input slices, and the resulting weighting matrices, $\mathbf{W}_{1}$ and $\mathbf{W}_{2}$ of FASTMER.

results without mis-registration are given Fig. 1.

It can be seen that the result of FASTMER converges to the result of TV + wavelet when a severe misregistration of $45^{\circ}$ exists. This is indeed what was expected: FASTMER ignores the reference due to major changes between the reference and the acquired image. Rotation of $5^{\circ}$ provides an improved reconstruction of PSNR $=32 \mathrm{~dB}$, while a minor rotation and a $5 \mathrm{~mm}$ translation provides results which are slightly better than the wavelet+TV reconstruction. The results of this experiment support the assumption described in the paper, that high degree of similarity with the reference image provides better results and vice versa. 
TABLE II FASTMER results at various degrees of similarity with the reference image

\begin{tabular}{|c|c|c|c|}
\hline $\begin{array}{c}\text { Misregistration } \\
\text { description }\end{array}$ & Image used as a reference & FASTMER results & $\begin{array}{c}\text { PSNR } \\
(\mathrm{dB})\end{array}$ \\
\hline Rotation of $5^{\circ}$ & & & 32 \\
\hline Rotation of $45^{\circ}$ & & & 28 \\
\hline $\begin{array}{c}\text { Rotation of } 5^{\circ}+ \\
\text { inter-plane } \\
\text { translation of } \\
5 \mathrm{~mm}\end{array}$ & & & 29 \\
\hline
\end{tabular}

\section{REFERENCES}

${ }^{1}$ Emmanuel J Candès. Compressive sampling. In Proceedings oh the International Congress of Mathematicians: Madrid, August 22-30, 2006: invited lectures, pages 1433-1452, 2006.

${ }^{2}$ D. L. Donoho. Compressed sensing. IEEE Transactions on Information Theory, 52(4): 1289-1306, 2006.

${ }^{3}$ Yonina C Eldar and Gitta Kutyniok. Compressed sensing: theory and applications. Cambridge University Press, 2012. 
${ }^{4}$ Yonina C Eldar. Sampling Theory: Beyond Bandlimited Systems. Cambridge University Press, 2015.

${ }^{5}$ Michael Lustig, David Donoho, and John M Pauly. Sparse MRI: The application of compressed sensing for rapid MR imaging. Magnetic resonance in medicine, 58(6):1182-1195, 2007.

${ }^{6}$ Shiqian Ma, Wotao Yin, Yin Zhang, and Amit Chakraborty. An efficient algorithm for compressed MR imaging using total variation and wavelets. In Computer Vision and Pattern Recognition, 2008. CVPR 2008. IEEE Conference on, pages 1-8. IEEE, 2008.

${ }^{7}$ Saiprasad Ravishankar and Yoram Bresler. MR image reconstruction from highly undersampled k-space data by dictionary learning. Medical Imaging, IEEE Transactions on, 30 (5):1028-1041, 2011.

${ }^{8}$ Xiaobo Qu, Di Guo, Bende Ning, Yingkun Hou, Yulan Lin, Shuhui Cai, and Zhong Chen. Undersampled MRI reconstruction with patch-based directional wavelets. Magnetic resonance imaging, 30(7):964-977, 2012.

${ }^{9}$ Jose Caballero, Anthony N Price, Daniel Rueckert, and Joseph V Hajnal. Dictionary learning and time sparsity for dynamic mr data reconstruction. Medical Imaging, IEEE Transactions on, 33(4):979-994, 2014.

${ }^{10}$ Zhi-Pei Liang and Paul C Lauterbur. A generalized series approach to MR spectroscopic imaging. IEEE Transactions on Medical Imaging, 10(2):132-137, 1991.

${ }^{11}$ Jill M Hanson, Zhi-Pei Liang, Erik C Wiener, and Paul C Lauterbur. Fast dynamic imaging using two reference images. Magnetic resonance in medicine, 36(1):172-175, 1996.

${ }^{12}$ Christopher P. Hess, Zhi-Pei Liang, and Paul C. Lauterbur. Maximum cross-entropy generalized series reconstruction. International Journal of Imaging Systems and Technology, 10(3):258-265, 1999.

${ }^{13}$ SungDae Yun, Sung Suk Oh, Yeji Han, and HyunWook Park. High-resolution fMRI with higher-order generalized series imaging and parallel imaging techniques (hgs-parallel). Journal of Magnetic Resonance Imaging, 29(4):924-936, 2009.

${ }^{14}$ Hien M Nguyen and Gary H Glover. A modified generalized series approach: application to sparsely sampled fMRI. IEEE Transactions on Biomedical Engineering, 60(10):2867-2877, 2013.

${ }^{15}$ Justin P Haldar, Van J Wedeen, Marzieh Nezamzadeh, Guangping Dai, Michael W Weiner, Norbert Schuff, and Zhi-Pei Liang. Improved diffusion imaging through SNR-enhancing 
joint reconstruction. Magnetic Resonance in Medicine, 69(1):277-289, 2013.

${ }^{16}$ Michael Lustig, Juan M Santos, David L Donoho, and John M Pauly. K-T sparse: High frame rate dynamic MRI exploiting spatio-temporal sparsity. In Proceedings of the 13th Annual Meeting of ISMRM, Seattle, volume 2420, 2006.

${ }^{17}$ Tao Lang and Jim Ji. Accelerating dynamic contrast-enhanced MRI using compressed sensing. In Proceedings of the 16th annual meeting of ISMRM, Toronto, Canada, page 1481, 2008.

${ }^{18}$ Urs Gamper, Peter Boesiger, and Sebastian Kozerke. Compressed sensing in dynamic MRI. Magnetic Resonance in Medicine, 59(2):365-373, 2008.

${ }^{19}$ Hong Jung, Kyunghyun Sung, Krishna S Nayak, Eung Yeop Kim, and Jong Chul Ye. k-t focuss: A general compressed sensing framework for high resolution dynamic MRI. Magnetic Resonance in Medicine, 61(1):103-116, 2009.

${ }^{20}$ Liyong Chen, Alexey Samsonov, and Edward VR DiBella. A framework for generalized reference image reconstruction methods including hypr-lr, pr-focuss, and k-t focuss. Journal of Magnetic Resonance Imaging, 34(2):403-412, 2011.

${ }^{21}$ Mark Chiew, Stephen M Smith, Peter J Koopmans, Nadine N Graedel, Thomas Blumensath, and Karla L Miller. k-t faster: Acceleration of functional MRI data acquisition using low rank constraints. Magnetic Resonance in Medicine, 2014.

${ }^{22}$ Ganesh Adluru, Yaniv Gur, Liyong Chen, David Feinberg, Jeffrey Anderson, and Edward VR DiBella. MRI reconstruction of multi-image acquisitions using a rank regularizer with data reordering. Medical physics, 42(8):4734-4744, 2015.

${ }^{23}$ Ricardo Otazo, Emmanuel Candès, and Daniel K Sodickson. Low-rank plus sparse matrix decomposition for accelerated dynamic MRI with separation of background and dynamic components. Magnetic Resonance in Medicine, 73(3):1125-1136, 2015.

${ }^{24}$ Bing Wu, Rick P Millane, Richard Watts, and Philip J Bones. Prior estimate-based compressed sensing in parallel MRI. Magnetic Resonance in Medicine, 65(1):83-95, 2011.

${ }^{25}$ Xi Peng, Hui-Qian Du, Fan Lam, S Derin Babacan, and Zhi-Pei Liang. Reference-driven MR image reconstruction with sparsity and support constraints. In 2011 IEEE International Symposium on Biomedical Imaging: From Nano to Macro, pages 89-92. IEEE, 2011.

${ }^{26}$ Huiqian Du and Fan Lam. Compressed sensing MR image reconstruction using a motioncompensated reference. Magnetic resonance imaging, 30(7):954-963, 2012. 
${ }^{27}$ Fan Lam, Justin P Haldar, and Zhi-Pei Liang. Motion compensation for referenceconstrained image reconstruction from limited data. In 2011 IEEE International Symposium on Biomedical Imaging: From Nano to Macro, pages 73-76. IEEE, 2011.

${ }^{28}$ Berkin Bilgic, Vivek K Goyal, and Elfar Adalsteinsson. Multi-contrast reconstruction with bayesian compressed sensing. Magnetic Resonance in Medicine, 66(6):1601-1615, 2011.

${ }^{29}$ Xiaobo Qu, Yingkun Hou, Fan Lam, Di Guo, and Zhong Chen. Magnetic resonance image reconstruction using similarities learnt from multi-modal images. In Signal and Information Processing (ChinaSIP), 2013 IEEE China Summit \& International Conference on, pages 264-268. IEEE, 2013.

${ }^{30}$ Xiaobo Qu, Yingkun Hou, Fan Lam, Di Guo, Jianhui Zhong, and Zhong Chen. Magnetic resonance image reconstruction from undersampled measurements using a patch-based nonlocal operator. Medical image analysis, 18(6):843-856, 2014.

${ }^{31}$ Junzhou Huang, Chen Chen, and Leon Axel. Fast multi-contrast MRI reconstruction. Magnetic resonance imaging, 32(10):1344-1352, 2014.

${ }^{32}$ Jeffrey Tsao, Babak Behnia, and Andrew G Webb. Unifying linear prior-informationdriven methods for accelerated image acquisition. Magnetic resonance in medicine, 46(4): 652-660, 2001.

${ }^{33}$ Alexey A Samsonov, Julia Velikina, John O Fleming, Mark L Schiebler, and Aaron S Filed. Accelerated serial MR imaging in multiple sclerosis using baseline scan information. In Proceedings of the 18th annual meeting of ISMRM, Stockholm, Sweden, page 4876, 2010.

${ }^{34}$ Guobin Li, Jürgen Hennig, Esther Raithel, Martin Büchert, Dominik Paul, Jan G Korvink, and Maxim Zaitsev. Incorporation of image data from a previous examination in $3 \mathrm{~d}$ serial MR imaging. Magnetic Resonance Materials in Physics, Biology and Medicine, pages 1-13, 2015.

${ }^{35}$ Xiaoping Hu, David N Levin, Paul C Lauterbur, and Thomas Spraggins. Slim: Spectral localization by imaging. Magnetic resonance in medicine, 8(3):314-322, 1988.

${ }^{36}$ Gene Gindi, Mindy Lee, Anand Rangarajan, et al. Bayesian reconstruction of functional images using anatomical information as priors. IEEE Transactions on Medical Imaging, 12(4):670-680, 1993.

${ }^{37}$ Xiaolong Ouyang, Wing H Wong, Valen E Johnson, Xiaoping Hu, and Chin-Tu Chen. Incorporation of correlated structural images in pet image reconstruction. IEEE Transactions on Medical Imaging, 13(4):627-640, 1994. 
${ }^{38}$ Sangeetha Somayajula, Christos Panagiotou, Anand Rangarajan, Quanzheng Li, Simon R Arridge, and Richard M Leahy. Pet image reconstruction using information theoretic anatomical priors. IEEE Transactions on Medical Imaging, 30(3):537-549, 2011.

${ }^{39}$ Guang-Hong Chen, Jie Tang, and Shuai Leng. Prior image constrained compressed sensing (piccs): a method to accurately reconstruct dynamic CT images from highly undersampled projection data sets. Medical physics, 35(2):660-663, 2008.

${ }^{40}$ Lior Weizman, Yonina C Eldar, and Dafna Ben Bashat. Compressed sensing for longitudinal MRI: An adaptive weighted approach. Medical Physics, 42(9), 2015.

${ }^{41} \mathrm{~S}$. Ravishankar and Y. Bresler. Adaptive sampling design for compressed sensing MRI. In 2011 Annual International Conference of the IEEE Engineering in Medicine and Biology Society, pages 3751-3755. IEEE, 2011.

${ }^{42}$ Lior Weizman, Ohad Rahamim, Roey Dekel, Yonina C Eldar, and Dafna Ben-Bashat. Exploiting similarity in adjacent slices for compressed sensing MRI. In 36th Annual International Conference of the IEEE Engineering in Medicine and Biology Society (EMBC), 2014, pages 1549-1552. IEEE, 2014.

${ }^{43}$ Lior Weizman, Yonina C Eldar, A Eilam, S Londner, M Artzi, and D Ben Bashat. Fast reference based MRI. In Engineering in Medicine and Biology Society (EMBC), 2015 37th Annual International Conference of the IEEE, pages 7486-7489. IEEE, 2015.

${ }^{44}$ Zhi-Pei Liang and Paul C Lauterbur. An efficient method for dynamic magnetic resonance imaging. IEEE Transactions on Medical Imaging, 13(4):677-686, 1994.

${ }^{45}$ Jeffrey Tsao, Peter Boesiger, and Klaas P Pruessmann. k-t BLAST and k-t sense: Dynamic MRI with high frame rate exploiting spatiotemporal correlations. Magnetic Resonance in Medicine, 50(5):1031-1042, 2003.

${ }^{46}$ Justin P Haldar, Diego Hernando, Sheng-Kwei Song, and Zhi-Pei Liang. Anatomically constrained reconstruction from noisy data. Magnetic Resonance in Medicine, 59(4):810$818,2008$.

${ }^{47}$ Emmanuel J Candès, Michael B Wakin, and Stephen P Boyd. Enhancing sparsity by reweighted 11 minimization. Journal of Fourier analysis and applications, 14(5-6):877905, 2008.

${ }^{48}$ Zhao Tan, Yonina C Eldar, Amir Beck, and Arye Nehorai. Smoothing and decomposition for analysis sparse recovery. IEEE Transactions on Signal Processing, 62(7):1762-1774, 2014 . 
${ }^{49}$ Jeffrey A Fessler and Bradley P Sutton. Nonuniform fast fourier transforms using min-max interpolation. Signal Processing, IEEE Transactions on, 51(2):560-574, 2003.

${ }^{50}$ Joao FC Mota, Lior Weizman, Nikos Deligiannis, Yonina C Eldar, and Miguel RD Rodrigues. Reference-based compressed sensing: A sample complexity approach. In 2016 IEEE International Conference on Acoustics, Speech and Signal Processing (ICASSP), pages 4687-4691. IEEE, 2016.

${ }^{51}$ Gary P Zientara, Lawrence P Panych, and Ferenc A Jolesz. Dynamically adaptive MRI with encoding by singular value decomposition. Magnetic Resonance in Medicine, 32(2): 268-274, 1994.

${ }^{52}$ Scott K Nagle and David N Levin. Multiple region MRI. Magnetic resonance in medicine, 41(4):774-786, 1999.

${ }^{53}$ Yun Gao and Stanley J Reeves. Optimal k-space sampling in MRSI for images with a limited region of support. Medical Imaging, IEEE Transactions on, 19(12):1168-1178, 2000 .

${ }^{54}$ Jarvis Haupt, Robert Nowak, and Rui Castro. Adaptive sensing for sparse signal recovery. In IEEE 13th Digital Signal Processing Workshop and 5th IEEE Signal Processing Education Workshop, 2009. DSP/SPE 2009., pages 702-707. IEEE, 2009.

${ }^{55}$ Jarvis Haupt, Rui M Castro, and Robert Nowak. Distilled sensing: Adaptive sampling for sparse detection and estimation. IEEE Transactions on Information Theory, 57(9): 6222-6235, 2011.

${ }^{56}$ Dennis Wei and Alfred O Hero. Multistage adaptive estimation of sparse signals. IEEE Journal of Selected Topics in Signal Processing, 7(5):783-796, 2013.

${ }^{57}$ Matthias Seeger, Hannes Nickisch, Rolf Pohmann, and Bernhard Schölkopf. Optimization of k-space trajectories for compressed sensing by bayesian experimental design. Magnetic resonance in medicine, 63(1):116-126, 2010. 